Electronic supplementary information for:

\title{
Macrophase-separated organic ionic plastic crystals/ PAMPS- based ionomer electrolyte: a new design perspective for flexible and highly conductive solid-state electrolytes
}

Nicolas Goujon, ${ }^{* a, b}$ Robert Kerra ${ }^{a}$ Charlotte Gervilliéa ${ }^{a}$ Yogita V. Oza ${ }^{a}$, Luke A. O’Della, Patrick C. Howletta and Maria Forsyth ${ }^{a, b}$

${ }^{a}$ Institute for Frontier Materials, Deakin University, Waurn Ponds, Victoria 3216, Australia.

E-mail: nicolas.goujon@polymat.eu

b Polymat, Institute for Polymer Materials, University of the Basque Country UPV/EHU, Joxe Mari Korta Center, Avda. Tolosa 72, 20018 Donostia-San Sebastian, Spain

\section{Table of content}

1. Additional differential scanning calorimetry results 


\section{Additional differential scanning calorimetry results}

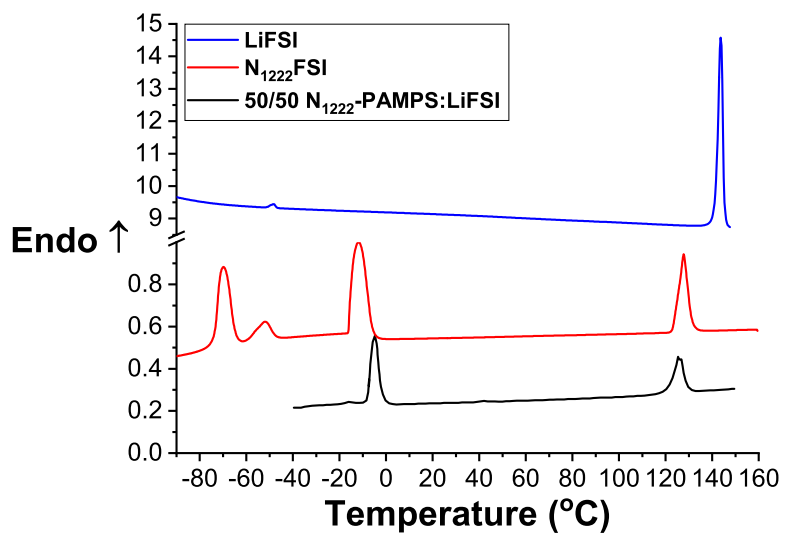

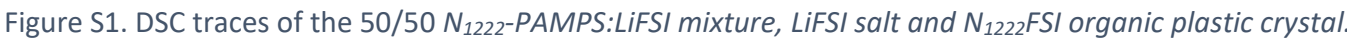



Figure S2.DSC trace of the 50/50 N1222-PAMPS:LiFSI mixture with an extended temperature range 